\title{
PRELIMINARY RESULTS ON LUMINAIRE DESIGNS FOR HYBRID SOLAR LIGHTING SYSTEMS
}

\author{
D. D. Earl, Oak Ridge National Laboratory \\ 2360 Cherahala Blvd. \\ Knoxville, TN 37932
}

\author{
J. D. Muhs, Oak Ridge National Laboratory \\ 2360 Cherahala Blvd. \\ Knoxville, TN 37932
}

\begin{abstract}
We report on the design of two hybrid lighting luminaires that blend light from a fiber optic end-emitted solar source with electric T8 fluorescent lamps. Both designs involve the retrofit of a commercially-available recessed fluorescent luminaire with minimal reductions in the original luminaire's optical efficiency. Two methods for high-angle dispersion of fiber optic endemitted solar light are described and the resulting spatial intensity distributions, simulated using ZEMAX, are compared with standard cylindrical fluorescent tubes. Differences in spatial intensity distribution are qualitatively characterized and potential design improvements discussed.
\end{abstract}

\section{INTRODUCTION}

We earlier reported a new hybrid solar lighting system which resulted in energy savings through direct utilization of visible solar light for internal lighting ${ }^{1,2}$, see Figure 1. A major step toward the realization of using fiber optic transported solar light for internal lighting purposes involves the development of a hybrid luminaire to seamlessly balance lamp and fiber optic transported solar illuminants. Fluctuations in the intensity of collected solar light, due to changing cloud coverage or solar collector movement, requires rapid compensation by electric lamps to maintain a constant room illumination. If the spatial intensity distribution of a hybrid luminaire's electric lamp does not closely match the spatial intensity distribution of the luminaire's fiber optic end-emitted solar illuminant, then the shift between artificial and solar lighting will be noticeable to the occupant and is highly undesirable.

To develop a hybrid luminaire that exhibits a static spatial intensity distribution regardless of which lighting source dominates, two light dispersing techniques were developed which permit fiber optic end-emitted light to mimic an elongated cylindrical source. Two common commercial fluorescent fixtures were selected and modified to incorporate the solar illuminant dispersing devices. The hybrid configurations were modeled using ZEMAX optical software and simulated under various conditions. The resulting spatial intensity differences between the electric and solar sources are presented in this paper. Chromaticity differences between the two sources are not addressed in this paper but will be presented in other publications.

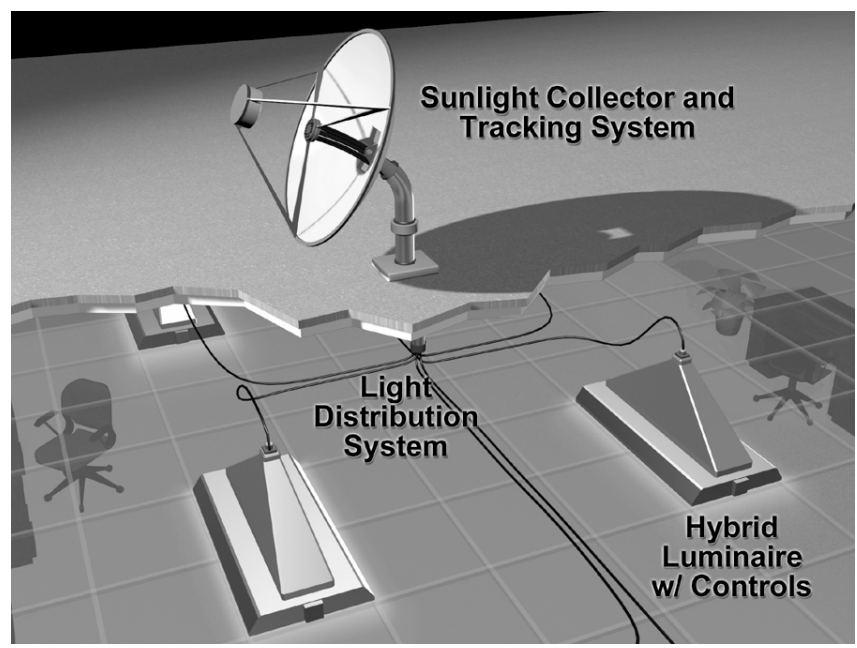

Figure 1. Hybrid Solar Lighting System

\section{SELECTION AND MODELING OF COMMERICIAL LUMINAIRE}

A wide variety of commercially available luminaires exist which can be adapted for use as hybrid luminaires. However, to limit our design effort, only two commercial luminaires were selected for retrofitting. The Lithonia General Purpose T8 Troffer and the Lithonia PARAMAX ${ }^{\circledR}$ Parabolic Troffer were chosen because of their high electrical efficiency, high optical 
efficiency (luminaire efficacy), relative low cost, and easily integrated design. Both troffers utilize four T8 lamps, are similar in dimension, and can be purchased with a variety of attached optical diffusing elements. A 32-cell, 24-cell, 18-cell, and 16-cell low iridescent anodized diffuse silver coated louvre baffle and \#19 pattern $4.0 \mathrm{~mm}$ thick acrylic lens diffuser were considered for use with both troffer styles. A high-detail optical model of each fixture combination was constructed using ZEMAX ray-tracing software and the optical efficiency and performance of the fixtures were simulated. The spatial intensity distribution of each fixture was simulated for ceiling placement within a six-foot cube. The top two design combinations, which produced a spatial intensity distribution that could be most easily mimicked by a fiber optic end-emitted solar source, were chosen for retrofitting. The simulated spatial intensity distribution for one such configuration is shown in Figure 2.

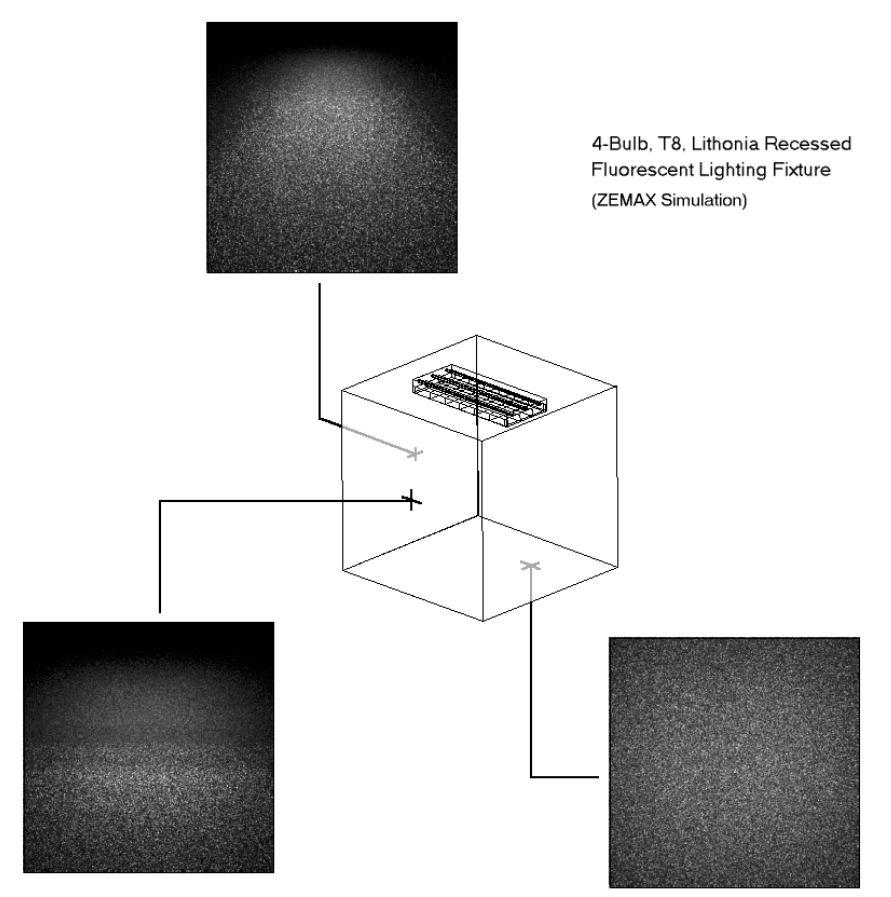

Figure 2. Spatial Intensity Distribution of Selected Commercial Fixture

As expected, the fixture in Figure 2 produces a pseudo-elliptical spatial intensity distribution. Light from the sides of the fixture is diffused across a larger area than light emitted from the back or front of the fixture. The elliptical nature of the fixture's diffused light represents one of the major technical challenges faced in efficiently converting radially-symmetric light into an extended cylindrical source.

\section{HYBRID LUMINAIRE USING CYLINDRICAL DIFFUSING ROD}

Seeking a direct approach toward transforming fiber optic end-emitted light into a cylindrical light source, the development of a cylindrical diffusing rod was pursued. The model for a cylindrical diffusing rod consisted of a $2.54 \mathrm{~cm}$ diameter, $1.0 \mathrm{~m}$ long, optically clear cylinder with a polished lower hemisphere and a diffuse upper hemisphere. Light launched from a butt-coupled optical fiber, scatters from the diffuse upper surface of the cylinder and escapes through the polished lower surface of the cylinder. To improve efficiency, upward-scattered light is redirected back toward the lower hemisphere of the diffusing rod with a silver-coating on the upper hemisphere.

Three diffusing rods, each placed mid-way and slightly above adjacent fluorescent lamps in a 4-tube PARAMAX ${ }^{\circledR}$ Parabolic Troffer with 24-cell louvre baffle, were expected to produce a spatial intensity distribution which closely matched that of the four fluorescent tubes. However, initial modeling of the diffusing rod indicated that the intensity of the scattered light was too highly concentrated toward one end of the rod, creating uneven illumination. In addition, a large portion of the light entering the diffusing rod at small angles was not being scattered at all and, instead, was merely being reflected from the planar end of the diffusing rod back into the butt-coupled optical fiber. To overcome these deficiencies, a silver-coated concave mirror surface at the end of the rod was added to the diffusing rod model, see Figure 3. This concave end-mirror strongly diverged low-angle incident light, hence improving the optical efficiency of the diffusing rod while also improving the overall uniformity of the scattered light. To further improve the uniformity of the scattered light, a $40 \mathrm{~cm}$ strip along the center of the diffusing rod's top hemisphere was modeled with a larger scattering fraction than the outer ends to increase the amount of scattered light emitted from the center of the diffusing rod.

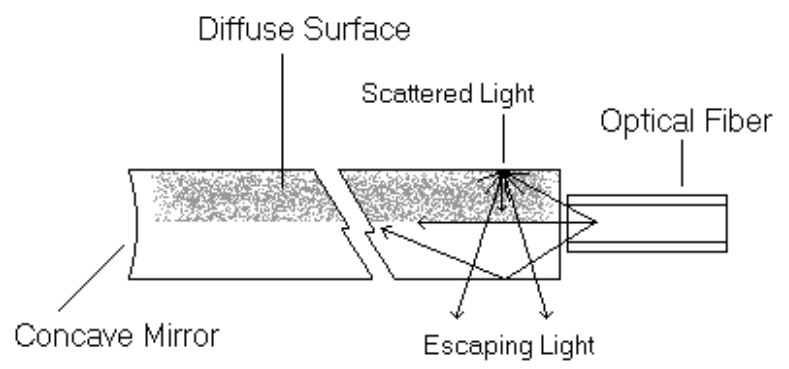

Figure 3. Cylindrical Diffusing Rod Design

Simulations of the spatial intensity distribution resulting from the fluorescent lamps and/or the diffusing rods revealed only minor differences between the two distributions, and only minor deviation from the fixture's original spatial intensity distribution. However, due to obstruction and scattering losses associated with the inclusion of the three diffusing rods, the optical efficiency of the fixture was decreased from $64 \%$ to 
53\%. The diffusing rod itself was estimated to be only $50 \%$ efficient at converting a fiber optic end-emitted source into a cylindrical source. This efficiency was strongly dependent upon the intensity profile of the fiber optic end-emitted light and the combination of scattering values used along the top surface of the diffusing rod.

To physically realize the cylindrical diffusing rod design, a $2.54 \mathrm{~cm}$ diameter, $1 \mathrm{~m}$ long, cast acrylic rod, with high optical clarity and optically smooth outer surface, was used. The rod was diamond-machined on one end to create a concave surface with a radius of curvature of $4.0 \mathrm{~cm}$, and polished on the other end to create a planar optical surface suitable for butt-coupling to a large-core optical fiber. The top hemisphere of the rod was sandblasted to produce a uniform scattering surface and both the top hemisphere and concave end-mirror were coated with aluminum. Due to construction limitations, the top surface did not exhibit a variable surface scatter as originally modeled.

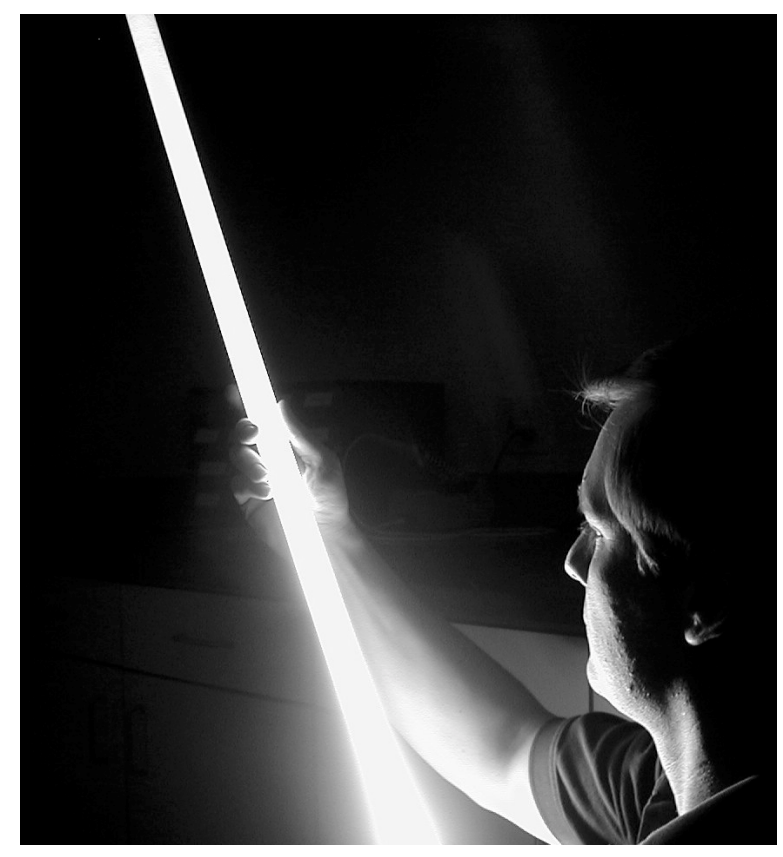

Figure 4. Constructed Cylindrical Diffusing Rod

Preliminary testing of the cylindrical diffusing rod revealed a discrepancy between the desired modeled surface scatter and the actual surface scatter created by the sandblasting technique. Because optical scattering is often difficult to accurately premodel in software, the result was not entirely unexpected. The actual surface scatter created by the sandblasting technique was much larger than modeled and created a diffusing rod with an uneven illumination, see Figure 4. However, now given the correlation between the modeled scattering values and the actual scattering values, it may be possible to re-simulate and re-design the cylindrical diffusing rod to emit a more uniform intensity distribution. Additional factors related to optical efficiency and construction costs are currently being evaluated.

\section{HYBRID LUMINAIRE USING MULTIPLE LIGHT DISPERSING ELEMENTS}

A four-tube Lithonia GT8 troffer equipped with a \#19 pattern $4.0 \mathrm{~mm}$ acrylic lens diffuser, which has been modified to include two $15 \mathrm{~cm}$ diameter proprietary light dispersing elements which utilize micro-optic structures, may offer the most promising design yet for a hybrid luminaire, see Figure 5.

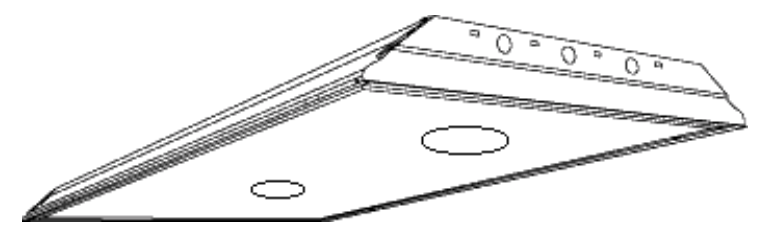

Figure 5. GT8 Hybrid Luminaire Design

The two $4.0 \mathrm{~mm}$ thick light dispersing elements are mounted within the fixture's acrylic lens diffuser and allow a range of highly divergent quasi-elliptical spatial intensity distributions to be generated from two circular fiber optic end-emitting sources fed through the top surface of the fixture's troffer. Variations in the spatial intensity distribution of the dispersed solar light can be made without the need to readjust the alignment or spacing between the optical fiber and the light dispersing element. A static configuration, similar to Figure 6 , is maintained.

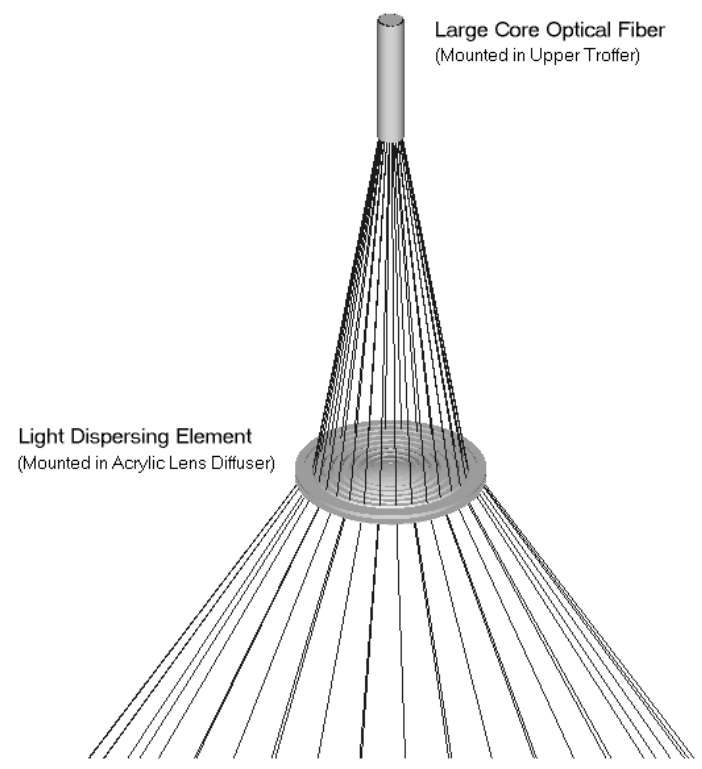

Figure 6. Typical Configuration of Light Dispersing Element and Optical Fiber

The optical efficiency of the light dispersing elements has been estimated at greater than $90 \%$ while the optical efficiency of the original luminaire is reduced by $3 \%$, from $76 \%$ to $73 \%$. To improve the matching between the electric and solar spatial intensity distributions, the number of elements can be increased 
to four or even eight. However, increasing the number of light dispersing elements reduces the optical efficiency of the fixture and results in higher costs. Construction and experimental verification of a two-element hybrid luminaire is underway.

\section{IMPROVEMENTS}

The two hybrid luminaires presented in this paper still require a significant amount of additional testing, modeling, and re-design before their physical implementations will realize their full potential. Improved scattering models are needed to more accurately describe the physical processes used in constructing the cylindrical diffusing rod while an improved model of micro-optic-based light dispersing elements still remains to be experimentally verified.

\section{ACKNOWLEDGMENTS}

Research at ORNL was sponsored by the Energy Efficiency and Renewable Energy Office of the U.S. Department of Energy and the Public Power Institute of the Tennessee Valley Authority.

\section{REFERENCES}

1. J. D. Muhs, "Hybrid Solar Lighting Doubles the Efficiency and Affordability of Solar Energy in Commercial Buildings", CADDET Energy Efficiency Newsletter December 2000, p. 6.

2. J. D. Muhs, "Design and Analysis of Hybrid Solar Lighting and Full-Spectrum Solar Energy Systems", Solar 2000, July 16-21, 2000, American Solar Energy Society. 